\section{Picture This: The State of the Art in Visualization for Complex Adaptive Systems}

\begin{abstract}
Visualization has an increasingly important role to play in scientific research. Moreover, visualization has a special role to play within artificial life as a result of the informal status of its key explananda: life and complexity. Both are poorly defined but apparently identifiable via raw inspection. Here we concentrate on how visualization techniques might allow us to move beyond this situation by facilitating increased understanding of the relationships between an ALife system's (low-level) composition and organization and its (high-level) behavior. We briefly review the use of visualization within artificial life, and point to some future developments represented by the articles collected within this special issue.
\end{abstract}

\author{
Seth Bullock* \\ School of Electronics and \\ Computer Science \\ University of Southampton \\ Southampton, SO17 1BJ, UK \\ sgb@ecs.soton.ac.uk

\section{Jon Bird} \\ Tom Smith \\ Department of Informatics \\ University of Sussex \\ Brighton, BN1 9QH, UK \\ toms@cogs.susx.ac.uk \\ jonba@sussex.ac.uk
}

Picture this, a sky full of thunder,

Picture this, my telephone number. .

— "Picture This" (Destri/Harry/Stein), Blondie, Parallel Lines, 1979

Understanding artificial life systems typically involves explicating the relationship between (at least) two levels of description. How does a list of "telephone numbers," formal structures, rules, codes, algorithms, and so on, give rise to complex, collective, "organic" behavior that can be confusingly nebulous to the point of impenetrability? In this special issue, we explore the role of visualization in helping to clarify these kinds of relationship.

As data sets have grown and models have become more complicated, data visualization has had an increasingly important role to play in science. Our ability to effectively and reliably represent systems visually, so that our most developed sensory mechanisms are afforded the maximum chance to identify interesting patterns, increases in significance with both the volume of data available and the complexity of the patterns within it.

Perhaps surprisingly, research into how complex adaptive systems might be most effectively visualized has not been a particularly active theme within artificial life or related disciplines. While the recent increase in affordable computing power (in terms of speed, storage, and graphics) has seen a growing interest in increasingly sophisticated visualization within the community, questions of visualization have not been the explicit focus of many articles or projects. Rather, visualization research of this kind has tended to take place tacitly as a kind of unrecognized scaffolding activity that is typically published in technical reports, if at all. Although a number of reviews of visualization

\footnotetext{
* Corresponding author.
} 
for evolutionary algorithms have been published $[12,3,7]$, this special issue seeks to highlight the wider arena of research into visualization for artificial life.

Within science in general, two very different uses of visualization can be identified. During initial exploratory research, multiple creative attempts to visualize data must typically be made before critical insight is achieved. At this stage, "private" visualizations are sometimes idiosyncratic, complicated, and informal. However, should this exploration lead to publishable results, a very different type of visualization is required in order to effectively disseminate them. In order to readily convey the aspects of the data that have been determined to be relevant, the constraints imposed by the format of the dissemination medium and the character of the audience must each be considered. Consequently, by the time research reaches the printed page there is pressure to employ a limited number of visualizations that are each simple, standardized, and hence intuitive. Simply transferring visualizations that were effective during one stage to the other is rarely successful. Indeed, inappropriate choices of visualization at either stage can severely compromise progress and intelligibility. Given how important these choices are, it is perhaps surprising that scientists generally receive little training in how to make them.

For artificial life researchers, however, the issue of visualization is even more acute, for at least two reasons related to ALife's objects of study. First, unlike most scientific visualization, which is concerned with finding ways of usefully reformatting or summarizing data sets, many products of ALife research are visualizations - here visual presentation realizes the system in the first place rather than re-presenting (i.e., recoding, transforming) it for visual analysis. Conway's game of life [11], Dawkins' biomorphs [8, 9], Reynolds' boids [18], and Sims' blockies [20] are well-known ALife systems where the visualization is the system under scrutiny. Given artificial life's aim of synthesizing lifelike systems, it should perhaps not be surprising that the scientific value of ALife systems is to some degree founded on the extent to which they appear lifelike to us. For as long as artificial life's key concern (whether or not a system can be said to be alive) remains an elusive "I'll know it when I see it" property, we will be interested in the raw subjective appearance of ALife systems and in the extent to which this appearance can (or cannot) be related to or explained by the system's constitution and organization.

A second and related issue concerns the apparent complexity of artificial life systems. Again, we currently lack standardized formal tools for identifying interesting complexity, often having to rely upon bringing our unaided cognitive capabilities to bear on a representation of as much of the system's behavior as we can squeeze onto the screen:

having myself seen thousands of pictures produced by cellular automata, I can recognize immediately from memory almost any pattern generated by any of the elementary rules even though none of the other methods of perception and analysis can get very far whenever such patterns are at all complex. - Stephen Wolfram [25, p. 621].

While our ability to extract useful information from such "raw" images (and to store vast amounts of it) is impressive, it remains mysterious and thus problematic. In order to understand the relationships between an ALife system's organization and its lifelike or complex behavior, we need to account for, rather than merely identify, the patterns of activity exhibited by these systems. Visualization of a more traditional (although not necessarily straightforward) nature is required in order to achieve this kind of objective - that is, visualization used as an analytic rather than merely descriptive tool.

This collection brings together a number of different approaches, moving beyond depictions of ALife towards the analytic representation of ALife abstractions-metrics and measures that track theoretically motivated systemic properties. ${ }^{1}$ In taking this approach, we are reflecting

I Within this special issue, Eldridge's article [10] is something of a departure in that it concentrates on the benefits to be gained from presenting systems via a novel sensory modality - hearing rather than vision. 
an ongoing trend within artificial life, which is increasingly concerned with ways of measuring and monitoring complex adaptive systems, rather than simply presenting them to the naked eye.

The proceedings of the first two international conferences on ALife $[13,14]$ are packed with screenshots, photographs, pseudocode, realistic and schematic line drawings, and pictures of artificial worlds. Many of these images are attempts to depict the (behavior of the) systems being presented. By contrast, the two most recent proceedings of the same conference series [22,17] are dominated by various forms of line graph and bar chart, schematic diagrams (mostly representing kinds of network), phase portraits, tables, and occasional 3D surface and contour plots. While photos (principally of physical robots or biological phenomena) and screenshots (in particular of 1D cellular automata's behavior, 2D gridworlds, and 3D virtual realities) are still employed, their usage has tended to be standardized and focused within particular subfields of research (e.g., autonomous robotics).

Over the last 15 years or so, then, there has been a winnowing of ALife visualization forms, with certain lineages dwindling (pseudocode, realistic line drawings), while others proliferate (e.g., line graphs depicting variation in some property over time). Moreover, over the same period, ALife has been responsible for originating a large number of novel visualizations. The vast majority of these idiosyncratic approaches appear to have been developed primarily in order to help their originator to understand a particular kind of ALife system rather than as a means of effectively conveying information to a wider audience. While understanding visualizations of this kind often requires some effort on the part of the reader, they can be useful in context even if they tend not to proliferate. However, ALife has witnessed the birth of a small number of successful visualization lineages, innovations that have been adopted by a number of subsequent researchers, some of which are detailed in the first four articles in this collection: four ALife visualization "gems" $[2,5,15,24]$. By contrast, the fifth contribution serves as a review of the manner in which standard techniques can be employed successfully [16]. The remaining four articles present active research in artificial life visualization: the use of deforming 2D maps to represent the evolution of structural relationships in a developing language [1]; a method of generating recognizable phylogenetic trees from evolutionary simulation model data [4]; the extent to which auditory representations are useful in conveying relevant properties of complex adaptive systems [10]; and graph techniques for representing evolutionary flows in a high-dimensional genetic space [19].

There is significant potential to build on these kinds of studies. However, there is also room for improvements in methodology, particularly in terms of the requirements for capture and formal evaluation. We currently have little appreciation of the different character of the various types of ALife practitioner (novices, experts, researchers, industrialists, tutors, students, etc.), and little idea of their needs and abilities (although see [6]). Compounding this lack of knowledge, we have little understanding of the usability or effectiveness of existing ALife visualization techniques (although see [23]). Further research attending to each of these aspects is urgently required if visualization is to help ALife move forward and address its stated aims.

\section{Acknowledgments}

This article and the special issue it introduces grew out of a workshop, "Beyond Fitness: Visualizing Evolution," that took place in 2002 at the Eighth International Conference on Artificial Life [21]. We thank the workshop contributors and attendees, Andy Philippides and Martin Thompson for assistance, and Mark Bedau for suggesting that a special issue of Artificial Life might be a suitable way of taking the ideas forward. We would also like to thank the authors who submitted contributions to this issue, the referees who reviewed them, and Simon Davy, who assisted with some careful proofreading.

Finally, thanks also to Hewlett-Packard Laboratories and British Telecom, which have kindly donated funds that enable us to include a set of color plates. 


\section{References}

1. Brighton, H., \& Kirby, S. (2006). Understanding linguistic evolution by visualizing the emergence of topographic mappings. Artificial Life, 12(2), 229-242.

2. Bullock, S., \& Bedau, M. (2006). Exploring the dynamics of adaptation with evolutionary activity plots. Artificial Life, 12(2), 193-197.

3. Bullock, S., Cartlidge, J., \& Thompson, M. (2002). Prospects for computational steering of evolutionary computation. In [21] (pp. 131-137).

4. Clement, R. (2006). Visualizing speciation in artificial cichlid fish. Artificial Life, 12(2), 243-257.

5. Cliff, D., \& Miller, G. F. (2006). Visualizing coevolution with CIAO plots. Artificial Life, 12(2), 199-202.

6. Collins, T. D. (1998). The application of software visualization technology to evolutionary computation. Ph.D. thesis, The Open University, Milton Keynes, UK.

7. Collins, T. D. (2003). Visualizing evolutionary computation. In A. Ghosh \& S. Tsutsui (Eds.), Advances in evolutionary computation: Theory and applications (pp. 95-116). New York: Springer.

8. Dawkins, R. (1986). The blind watchmaker. New York: W. W. Norton.

9. Dawkins, R. (1989). The evolution of evolvability. In [13] (pp. 201-220).

10. Eldridge, A. (2006). Issues in auditory display. Artificial Life, 12(2), 259-274.

11. Gardner, M. (1970). The fantastic combinations of John Conway's new solitaire game "Life." Scientific American, 223(October), 120-123.

12. Hart, E., \& Ross, P. (2001). GAVEL: A new tool for genetic algorithm visualization. IEEE Transactions on Evolutionary Computation, 5(4), 335-348.

13. Langton C. G. (Ed.) (1989). Artificial Life: Proceedings of the First Interdisciplinary Workshop on the Synthesis and Simulation of Living Systems. Redwood City, CA: Addison-Wesley.

14. Langton, C. G., Taylor, C., Farmer, J. D., \& Rasmussen, S. (Eds.) (1992). Artificial Life II: Proceedings of the Second International Conference on Artificial Life. Redwood City, CA: Addison-Wesley.

15. Pohlheim, H. (2006). Multidimensional scaling for evolutionary algorithms: Visualization of the path through search space and solution space using Sammon mapping. Artificial Life, 12(2), 203-209.

16. Pohlheim, H. (2006). Understanding the course and state of evolutionary optimizations using visualization-Ten years of industry experience with evolutionary algorithms. Artificial Life, 12(2), $217-227$.

17. Pollack, J., Bedau, M., Husbands, P., Ikegami, T., \& Watson, R. A. (Eds.) (2005). Artificial Life IX: Proceedings of the Ninth International Conference on Artificial Life. Cambridge, MA: MIT Press.

18. Reynolds, C. W. (1987). Flocks, herds, and schools: A distributed behavioral model. Computer Graphics, 21(4), $25-34$.

19. Salzberg, C., Antony, A., \& Sayama, H. (2006). Visualizing evolutionary dynamics of self-replicators: A graph-based approach. Artificial Life, 12(2), 275-287.

20. Sims, K. (1995). Evolving 3D morphology and behavior by competition. Artificial Life, 1, 353-372.

21. Smith, T., Bullock, S., \& Bird, J. (2002). Workshop overview. In T. Smith, S. Bullock, \& J. Bird (Eds.), Beyond Fitness: Visualizing Evolution-Workshop Proceedings of the Eighth International Conference on Artificial Life (pp. 99-102). University of New South Wales, Australia.

22. Standish, R., Bedau, M., \& Abbass, H. (Eds.) (2002). Artificial Life VIII: Proceedings of the Eighth International Conference on Artificial Life. Cambridge, MA: MIT Press.

23. Wiles, J., \& Tonkes, B. (2002). Visualisation of hierarchical cost surfaces for evolutionary computation. In D. Fogel (Ed.), Congress on Evolutionary Computation (pp. 157-162). Piscataway, NJ: IEEE Press.

24. Wiles, J., \& Tonkes, B. (2006). Hyperspace geography: Visualizing fitness landscapes beyond 4D. Artificial Life, 12(2), 211-216.

25. Wolfram, S. (2002). A new kind of science. Champaign, IL: Wolfram Media Inc. 